\title{
Nuclear Data Uncertainties for Typical LWR Fuel Assemblies and a Simple Reactor Core
}

\author{
D. Rochman, ${ }^{1}$ * 0. Leray, ${ }^{1}$ M. Hursin, ${ }^{1}$ H. Ferroukhi, ${ }^{1}$ A. Vasiliev, ${ }^{1}$ A. Aures, ${ }^{2}$ F. Bostelmann, ${ }^{2}$ W. Zwermann, ${ }^{2}$ 0. Cabellos, ${ }^{3}$ \\ C.J. Diez, ${ }^{3}$ J. Dyrda, ${ }^{3}$ N. Garcia-Herranz, ${ }^{4}$ E. Castro, ${ }^{4}$ S. van der Marck, ${ }^{5}$ H. Sjostrand, ${ }^{6}$ A. Hernandez, ${ }^{6}$ M. Fleming, ${ }^{7}$ J.-Ch. \\ Sublet, $^{7}$ and L. Fiorito ${ }^{8}$ \\ ${ }^{1}$ Reactor Physics and Systems Behaviour Laboratory, Paul Scherrer Institut \{PSD, 5232 Villigen Switzerland, ${ }^{2}$ Gesellschaft for \\ Anlagen- und Reaktorsicherhe it \{GRS\}, 85748 Garching, Germany, ${ }^{3}$ OECD Nuclear Energy Agency (NEA), Issy-les-Moulineaux, \\ France, ${ }^{4}$ Unwersidad Politecnica de Madrid \{UPM\}, Madrid, Spain, ${ }^{5}$ Nuclear Research and Consultancy Group \{NRG\}, Petten, The \\ Netherlands, ${ }^{6}$ Uppsala University, Department of Physics and Astronomy, Uppsala, Sweden, ${ }^{7}$ United Kingdom Atomic Energy \\ Authority \{UKAEA\}, Abingdon, UK, ${ }^{8}$ Institute for Advanced Nuclear Systems, SCK・GEN, Belgium
}

\section{A B S T R A C T.}

The impact of the current nuclear data library covariances such as in ENDF/B-VII.1, JEFF- 3.2, JENDL-4.0, SCALE and TENDL, for relevant current reactors is presented in this work. The uncertainties due to nuclear data are calculated for existing P $\backslash . V R$ and BWR fuel assemblies (with burn-up up to $40 \mathrm{GWd} / \mathrm{tHM}$, followed by 10 years of cooling time) and for a simplified PWR full core model (without burn-up) for quantities such as k $\infty$, macroscopic cross sections, pin power or isotope inventory. In this work, the method of propagation of uncertainties is based on random sampling of nuclear data, either from covariance files or directly from basic parameters. Additionally, possible biases on calculated quantities are investigated such as the self-shielding treatment. Different calculation schemes are used, based on CASMO, SCALE, DRAGON, MCNP or FISPACT-11, thus simulating real-life assignments for technical-support organizations. The outcome of such a study is a comparison of uncertainties with two consequences. One: although this study is not expected to lead to similar results between the involved calculation schemes, it provides an insight on what can happen when calculating uncertainties and allows to give some perspectives on the range of validity on these uncertainties. Two: it allows to dress a picture of the state of the knowledge as of today, using existing nuclear data library covariances and current methods.

\section{O N T E N TS.}

\section{INTRODUCTION}

II.

A. Uncertainty Propagation Methods

1. PSI-SHARKX

2. PSI-SHARKX Fission Yields

3. GRS-XSUSA

4. UPM-SCALE

5. NRG-Fast TMC

6. UU-TMC

7. UKAEA-FISPACT-11

B.

8. SCK Fission Yields

Considered Systems
1. PWR UO2 Assembly

2. PWR UO2+Gd Assembly

3. PWR MOX Assembly

4. BWR UO2+Gd Assembly

5. BWR MOX+Gd Assembly

6. Martin-Hoogenboom Benchmark

C. Calculated Quantities

III. NUCLEAR DATA
B. Isotopes and Reactions $\quad 29$

* Corresponding author: dimitrialexandre rocbman@psi.ch 
C. Processing 31

1. PREPRO 31

2. CALENDF

3. NJOY for Covariances

4. AMPX

D. Missing Data

\section{RESULTS}

A. Assemblies $\mathrm{k}_{\infty}$

B. Assemblies Number Densities

C. Assemblies Two-group Cross Sections

D. Assemblies Pin Power Distribution

E. $\mathrm{k}_{\infty}$ Partial Contributors

F. Impact of Fission Yields
1. On $\mathrm{k}_{\infty}$
2. On Inventory
3. On Inventory (TMC Comparison)

G. Convergence, Correlation and Skewness

1. Convergence

2. Correlation

3. Skewness

H. Full Core - Power Distribution

1. Preliminary Tests

2. Tally F7

3. Tally F6

4. Comparison with the Fast GRS Method

I. Other Sources of Uncertainties

1. Operating Conditions

2. Manufacturing Tolerances

3. Burnup-induced Technological Changes

4. Computational Biases

5. Example

J. Self-shielding Treatment

1. Reactivity Coefficient UQ

2. Handling the Implicit Effect in SHARK-X 51

3. UQ Results

4. Conclusion for the Implicit Effect

\section{CONCLUSIONS AND OUTLOOK}

Acknowledgments

References

A. Additional Results on Uncertainties

\section{INTRODUCTION}

The simulation of the neutronic behavior of a reactor core is crucial for its safety. It intervenes at each stage of the life of a reactor: design, loading core licensing, cycle calculations, and dismantling. It also influences the fate of irradiated fuel: design, burn-up, transport, eventually reprocessing and finally storage. At the start of these simulations are the nuclear data (e.g. microscopic cross sections, angular and energy distributions for emitted particles, fission yields), and also their degree of knowledge (uncertainties, or covariances). One of the recurrent question is "how important are nowadays the nuclear data ?" In other terms: are they known enough, or do we (still) need to improve their precision with new measurements or new theoretical models. The answer is certainly not simple and depends on the field of application. For instance it is widely accepted that capture cross sections have an important impact in astrophysics (see for instance Ref. [1]). But the isotopes involved for such calculations may not exist in nuclear energy applications. Also, specific $(p, n)$ cross sections are of high importance for the production of new positron emission tomography (PET) tracers [2]; these reactions are nevertheless not common in energy applications. As a final example outside nuclear energy applications, neutron-induced single event effects in modern microelectronics can have a large impact for planes, space shuttles and the International Space Station, but the neutron energy range for damage is often above $10 \mathrm{MeV}[3]$.

In nuclear energy applications the question of the relevance of nuclear data needs to be answered in unequivocal terms for the safety of nuclear operations: yes the knowledge of nuclear data is enough for the usual operations of current nuclear reactors; and no it is not enough for their simulations outside the very restrictive limits related to their normal use. This apparent contradiction is based on the fact that the drive by experience is more reliable than the drive by fundamental understanding for thermal reactors. Additionally, the simulation tools have often been adjusted to achieve good performances in their validation limits, for instance by using adjusted nuclear data libraries. The consequence is that it becomes difficult to predict system behaviors with enough accuracy in the following areas:

- Outside the usual reactor operations,

- In typical fuel inventory,

- In local power distribution in the core,

- In dosimetry and neutron source predictions,

- In ex-core calculations,

- Outside neutronics code applicability (e.g. new reactor designs),

- Improvement of safety margins for cost reduction, 
- Higher fuel burn-up.

For additional information, the following references can be read as examples of some simulations compared to experiments: [4-6]. The improvement of predictions in the above fields does not automatically imply a nuclear data improvement. If the propagation of nuclear data uncertainty certainly indicates the need of better basic nuclear data measurements and theories, two other aspects can be improved: (1) better handling of nuclear data with improved processing codes, and (2) improvement of simulations by using more exact transport and solver systems. If these two points are not the subject of this paper, they should still be mentioned for completeness:

1. So-called "processing" of nuclear data allows to provide ready-to-use sets of data for simulations. The fact that the vast majority (with the exception of the AMPX code [7]) of such processing systems rely on (parts of) a single code such as NJOY [8] does not follow the principle of redundancy, often mandatory in nuclear environments. There is a very little significance for funding agencies to better measure quantities that NJOY can not handle, even if they can improve simulations (e.g. fission neutron emission as a function of fragment mass).

2. Neutronics simulation codes (transport, depletion, activation) as used as reference in the nuclear industry are often designed to compensate existing nuclear data inaccuracy. Such codes can therefore poorly handle "more correct" nuclear data as they will then provide deteriorated $\mathrm{C} / \mathrm{E}$ ratios (calculation over experiment ratios). But it should be noticed that Monte Carlo reference calculations can nowadays be used for the estimation of numerical biases.

Once we are aware of the current limitations of the calculation capabilities, it is however important to assess to which degree of accuracy such important quantities as nuclear data can provide. This is the first goal of this paper: presenting in a single study calculated uncertainties due to nuclear data for relevant systems in nuclear energy production. To fulfill this goal, different institutes agreed to participate, with their own method and database (see Sec. II A for the different definitions of methods). The only common points in these studies are (a) the systems (see Sec. IIB) and (b) the quantities to calculate (see Sec. II C).

Such an approach is definitively different compared to the Expert Group on Uncertainty Analysis in Modelling (UAM [9]). Here, because the libraries (both for nominal values and for covariances) are different, the calculated uncertainties can not be the same (see Sec. IV for results). The use of recommended covariance libraries is very convenient for the comparison and understanding of the various results, but it also restricts the possible spread of calculated responses due to the differences in the various covariance data libraries available. This difference of approach allows to simulate what happens in "real-life" assignments, where systems uncertainties are provided to another party. This is the second goal of this paper: providing a perspective on the relevance of uncertainties due to nuclear data, because the uncertainties due to nuclear data might not only be contained in its covariance files.

Finally, for nuclear simulations at the assembly or core level, nuclear data are not the only source of uncertainties. Even if it is not the main subject of this paper, a few indications are provided in Sec. IV I and IV J for the other potential effects on calculated quantities.

In a broader overview, the question of the uncertainties obtained from nuclear data with respect to specific requirements from the industry and safety authorities is a key issue. Such requirements might be different than the calculated uncertainties, as presented in Ref. [10]. The present paper does not touch on this subject, and further information can be obtained on the cited reference. In the case of current light water reactors, specific studies for the uncertainties on targeted quantities would be required and are not included here.

The present paper also does not intend to present results to benchmark a specific nuclear data library, as performed in Ref. [11]. For this intention, only specific and well dedicated efforts are necessary as it was realized for JENDL-4.0 [11]. Finally, the question of the feedback to improve nuclear data libraries based on benchmark results and sensitivities is not discussed here.

\section{METHODS}

The methods to calculate reactor quantities and their uncertainties differ because of the use of different transport codes, base libraries, covariance libraries and energy groups. A base library is a library which is used to calculate the nominal values, such as JEFF, ENDF/B, or JENDL [12-14]. It can differ from the sources of covariance information, for many practical reasons. Table I summarizes the main characteristics of each method used in this paper, depending on where the calculations are performed.

As presented in this table, the methods to calculate the uncertainties differ between the participants. More details are given in the following subsections.

\section{A. Uncertainty Propagation Methods}

\section{PSI-SHARKX}

At PSI, a methodology for the propagation of nuclear data covariance information is developed since 2011 [15, 20-22]. Since then, it was applied to many different burn-up calculations, see for instance Refs. [23, 24]. The SHARK-X methodology is based on the covariance files as provided in existing nuclear data libraries, and on a modified version of the deterministic lattice code 
TABLE I. Summary of the methods used to calculate uncertainties on the different quantities.

\begin{tabular}{|c|c|c|c|c|c|c|}
\hline Institute & $\overline{\text { PSI }}$ & $\overline{\text { GRS }}$ & UU & UPM & $\overline{\text { UKAEA }}$ & NRG \\
\hline Method name & SHARK-X & XSUSA & "Monte Carlo & $\overline{\text { SAMPLER }}$ & - & Fast-TMC \\
\hline Code & CASMO-5 & TRITON & DRAGON-5 & TRITON & FISPACT-II & MCNP6 \\
\hline Base library & ENDF/B-VII.0 & ENDF /B-VII.0 & JENDL-3.3 & ENDF/B-VII.1 & ENDF/B-VII.1 & ENDF/B-VII.1 \\
\hline Covariance & ENDF/B-VII.1 & SCALE-6.1 & JENDL-4.0 & SCALE-6.2beta5 & $\begin{array}{l}\text { TENDL-2015 } \\
\text { (fast range) }\end{array}$ & TENDL-2014 \\
\hline library & $\begin{array}{c}\text { SCALE-6.2beta4 } \\
\text { JEFF-3.2 } \\
\text { TENDL-2014 }\end{array}$ & & & & & \\
\hline Covariance FY & Many & - & - & $\begin{array}{c}\text { ENDF/B-VII.1 } \\
+ \text { SAMPLER } \\
{ }^{235} \text { U correlation }\end{array}$ & TENDL-2015 & TENDL-2014 \\
\hline Covariance groups & $\begin{array}{c}44 \\
56 \text { for SCALE-6.2 } \\
\end{array}$ & $\overline{44}$ & 69 & 56 & & pointwise \\
\hline Processing & NJOY2012 & AMPX & NJOY99 & AMPX & $\begin{array}{c}\text { NJOY2012 } \\
\text { PREPRO2015 } \\
\text { CALENDF2010 }\end{array}$ & NJOY99 \\
\hline Samples & 500 & 1000 & 300 & 200 & 500 & 500 \\
\hline Main reference & [15] & [16] & - & [17] & {$[18]$} & [19] \\
\hline
\end{tabular}

\section{CASMO-5M.}

\section{Modified CASMO-5}

CASMO-5 is a 2-dimensional deterministic fuel assembly code developed by Studsviks Scanpower [25]. It is delivered with its own processed library in binary format, which include cross sections, self-shielding factors, resonance integrals and other quantities. In its later versions, this library is based on ENDF/B-VII versions [12, 26], but also include other nuclear data evaluations from other libraries such as TENDL [27]. The processing of the original nuclear data evaluations into the library used by CASMO-5 is performed with NJOY, together with additional proprietary modules for specific formatting. It is therefore not possible to conveniently produce a different library version for CASMO-5, as it was performed for instance for DRAGON [28]. As a consequence, the CASMO-5 version was modified to allow for the alteration of some nuclear data while they are being used. This modified version of CASMO-5 used in SHARK$\mathrm{X}$ is called CASMO-5MX. The layout of the modified CASMO-5 code flow is presented in Fig. 1.

Self-shielding factors

The new modules allowing for the modifications of the nuclear data are indicated in red. In Fig. 1, the calculation flow of CASMO-5 shows that first a resonance calculation is performed for a representative set of pins (e.g. average, burnable absorbers). The resonance calculation step, which produces a set of resonance self-shielded microscopic cross section data, is performed in the resonance range. In the entire energy range, the step called "Microscopic cross section" produces a set of infinitely dilute cross sections (interpolated at the right temperature). These infinitely dilute cross sections are later replaced by the "PERTXS" module. It means that the self-shielding factors are not perturbed, even though by definition it should change given a new microscopic cross section.

This effect is known as the "implicit effect" and has been shown to be small for $\mathrm{k}_{\infty}$ uncertainty and two-group macroscopic cross section uncertainty [29]. In Ref. [29], the implicit effect is further analyzed in terms of the "direct effect" (directly due to the cross section perturbation) and the "indirect effect" (due to the induced change in the neutron flux). In the resonance range, it appears that the large increase (direct effect) is almost entirely compensated by a large decrease in the flux (indirect effect). So although this is a limitation of the current SHARK-X implementation, it is believed that the implicit effect is small, at least for responses which are not highly influenced by energy ranges including large resonances.

\section{PSI-SHARKX Fission Yields}

Fission yields can be randomly varied in the SHARK-X method as the other nuclear data quantities. The variations are also based on specific fission yield covariance matrices. The CASMO-5 neutron data libraries (ENDF/BVII.0, ENDF/B-VII.1 or JENDL 4.0) contain fission yield data for 27 fissioning species. The code does not contain all isotope decay chains (a limited set of 296 isotopes are considered in the version 5 for ${ }^{235} \mathrm{U}$ ) and therefore some approximations are made to limit the number of variables to handle. The included fission yields in the CASMO-5 database are either independent, cumulative or a combination of both, depending on the mass and nuclear charge of the considered fission fragment. Nuclides that are on the southeast edge of the CASMO-5 depletion chains are 


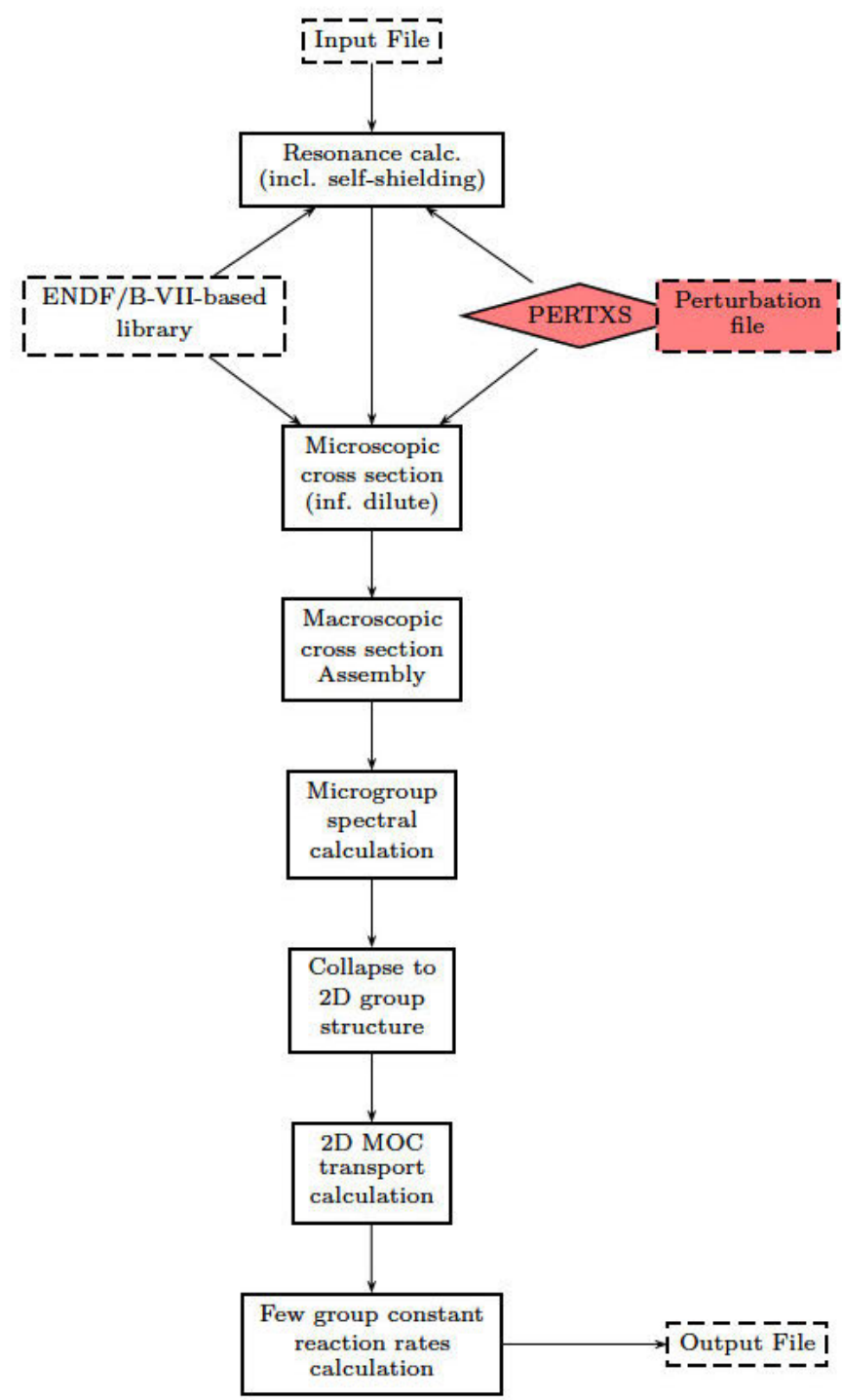

FIG. 1. (Color online) Work flow of the modified CASMO5MX code. The "red" boxes represent the added modules to CASMO-5M in order to modify the nuclear data.

represented with cumulative yield, whereas other nuclides are represented with an independent yield. The cumulative yield is approximately the sum of independent yields along the mass diagonal.

Two possible cases exist for the cumulative yield if both ground and meta-states exist for a particular ident. The cumulative yield for the ground state in the evaluated fission product library includes isomeric transition (IT) from the meta-states. The amount of IT is then computed from the branching ratio given in the radioactive decay data library.

- If both ground and one meta-state exist in the
CASMO-5 fission product chains, then the cumulative yield is given separately for each state. The amount of IT from the meta-state to the ground state must, however, be subtracted from the cumulative yield for the ground state before use in CASMO-5 (IT is included in the fission product chains).

- If the ground and meta-states are combined to a single ident in the CASMO-5 fission product chains then the cumulative yield for the combined ident is obtained by summing the cumulative yields for the two states and subtracting the amount of IT from the meta to the ground state.

The propagation of fission yield (FY) uncertainty through neutronics codes is non-trivial because fission yields constitute a very constrained system [30] and the following physical constraints must thus be respected. The following conservation equations can be considered, depending on the selected approach. The sum of independent FY from a given father (no ternary fission considered) is given by Eq. (1)

$$
\sum_{Z, A} Y(A, Z)=2
$$

with $Z, A$ the nuclear charge and the mass of the fission product, $Y$ its independent fission yield. The mass conservation (no spontaneous fission considered) implies that

$$
\sum_{Z, A} A \times Y(A, Z)=A_{\text {father }}+1-\nu_{p},
$$

with $A_{\text {father }}$ the mass of the target nucleus and $\nu_{p}$ the number of prompt emitted neutron per fission. The charge conservation implies that

$$
\sum_{Z, A} Z \times Y(A, Z)=Z_{\text {father }},
$$

with $Z_{\text {father }}$ the charge of the target nucleus, and finally, for every nuclear charge of the fission products $Z$, there exists a $Z$-complementary fission product

$$
\sum_{A} Y(A, Z)=\sum_{A} Y\left(Z_{\text {father }}-Z, A\right) .
$$

A consequence of these constraints is the difficulty to perturb FYs related to the evaluated fission yields: there are very simple covariance matrices available (only diagonal terms). These matrices do not contain correlation terms, neither for the independent nor cumulative fission yields. Additionally, the evaluated cumulative yields can be inconsistent with the independent yields, for both the nominal values and their uncertainties. Therefore, when performing stochastic sampling, different possibilities exist. In the current version of SHARK-X, four types of fission yield uncertainty propagation can be applied. In each case, all FYs are perturbed and an important number of code runs (e.g. 500-1000 samples) is therefore required in order to obtain trustworthy statistics. The four sampling types are: 
1. Sampling (according to the provided standard deviations) and perturbation without any constraints between the FYs (no constraints are respected). These direct perturbations can result in creating more fission products and inducing a bias on the output (e.g. reactivity, compositions),

2. Sampling without any constraints but with a normalization to 2 done after the sampling to fulfill the first condition (Eq. (1)). Consequently, the standard deviations of the samples change with the normalization process.

3. Sampling of the FYs using a constraint matrix as correlation matrix. The building of this matrix is explained in the next subsection.

An alternative approach is becoming nowadays possible with the help of theoretical codes such as GEF [31]. The GEF code allows to calculate among other quantities independent fission yields, which are globally in good agreements with current evaluations and selected experimental data. GEF is based on physical models and its parameters are adjusted to reproduce such data (one single set of parameters for all considered fissioning systems). The production of fission yield covariance files with GEF is based on the combination with a Bayesian Monte Carlo procedure (BMC), as presented in Ref. [32] for cross section evaluations and in Ref. [33] for fission yields. The succinct description is as follows and presented in Fig. 2:

1. Select models and parameter distributions from GEF,

2. Produce a set of random calculated quantities by sampling the parameter distributions,

3. Compare theoretical calculations and selected reference data, for instance with the $\chi^{2}$ quantity,

4. Use weights to update the parameter distributions,

5. Sample again to produce new calculated quantities based on the new weighted parameter distributions,

6. Repeat (3) to (5) until convergence of the parameter distributions.

Some details for the above steps are described below. There is fundamentally no difference with Ref. [32], except in the step (3) for the choice of the reference data. The set of reference data is chosen here to be the evaluated fission yields as given in the ENDF/B-VII.1 library. A list of the 21 most-important parameters for the GEF code is considered in this work, as obtained from Ref. [34] (see Table 7 in this reference) and is presented in Table II.

The adjustment of parameters and their updates is performed with a simplified $\chi^{2}$ goodness of fit-estimator. After $n$ iterations, the calculated GEF fission yields and uncertainties are close to the reference data (ENDF/BVII.1) and a correlation matrix between all fission products can be obtained. Examples are presented in Ref. [33] and an example of the correlation matrix is reproduced here for the thermal neutron-induced fission of ${ }^{235} \mathrm{U}$ (Fig. 3). The fission yields are ordered by sets of fission products in increasing masses, and by complementary couples (the nuclear charges of the two fragments sum to the nuclear charge of the fissioning system). For instance in the case of ${ }^{235} \mathrm{U}$, the plotted isotopes are all Ge, followed by $\mathrm{Nd}$ (presented in Fig. 3 by Ge/Nd), then $\mathrm{As} / \mathrm{Pr}, \mathrm{Se} / \mathrm{Ce}, \mathrm{Br} / \mathrm{La}, \ldots$ up to Tc/In. In total the correlation between about 250 fission yields are presented.

This defines the different possibilities in SHARK-X, which will be used and presented in section IV F.

\section{GRS-XSUSA}

The XSUSA ("Cross Section Uncertainty and Sensitivity Analysis") method is based on the random sampling GRS method (Gesellschaft für Anlagen- und Reaktorsicherheit) implemented in the code package SUSA "Software for Uncertainty and Sensitivity Analysis") [35]. The probability distributions of the uncertain input parameters are used to generate random variations of these input quantities. When applying this method with neutron cross section uncertainties, this means that many nuclear data libraries are generated, where all quantities with available uncertainties, namely inelastic and elastic scattering, (n,2n) and capture cross sections, in the case of fissionable nuclides additionally the fission cross section, the number of neutrons per fission, and the fission neutron spectrum, are varied at the same time for a large number of nuclides. As basis for generating the data variations, the SCALE 6.1 covariance data library is currently being used. This library contains uncertainties for relevant nuclides on the basis of various sources, including high-fidelity evaluations from ENDF/B-VII, ENDF/BVI, and JENDL-3.3, as well as approximate uncertainties obtained from a collaborative project performed by Brookhaven National Laboratory, Los Alamos National Laboratory, and Oak Ridge National Laboratory [36]. These covariance matrices are processed in a multi-group structure with 44 energy groups. When performing depletion calculations with the TRITON sequence from the SCALE 6.1 package [37], the default 94 nuclides in the fuel are taken into account. Thus, while the "traditional" SUSA method is predominantly being applied to problems with a limited number of parameters and only few correlations between them, the application to depletion calculations with nuclear data uncertainties leads to a large amount of uncertain parameters (94 nuclides, typically 2-8 reactions per nuclide, 44 energy groups per reaction), with a large amount of correlations between the energy group data of each nuclide/reaction combination, and also cross correlations between data of different reactions or even data for different nuclides. 


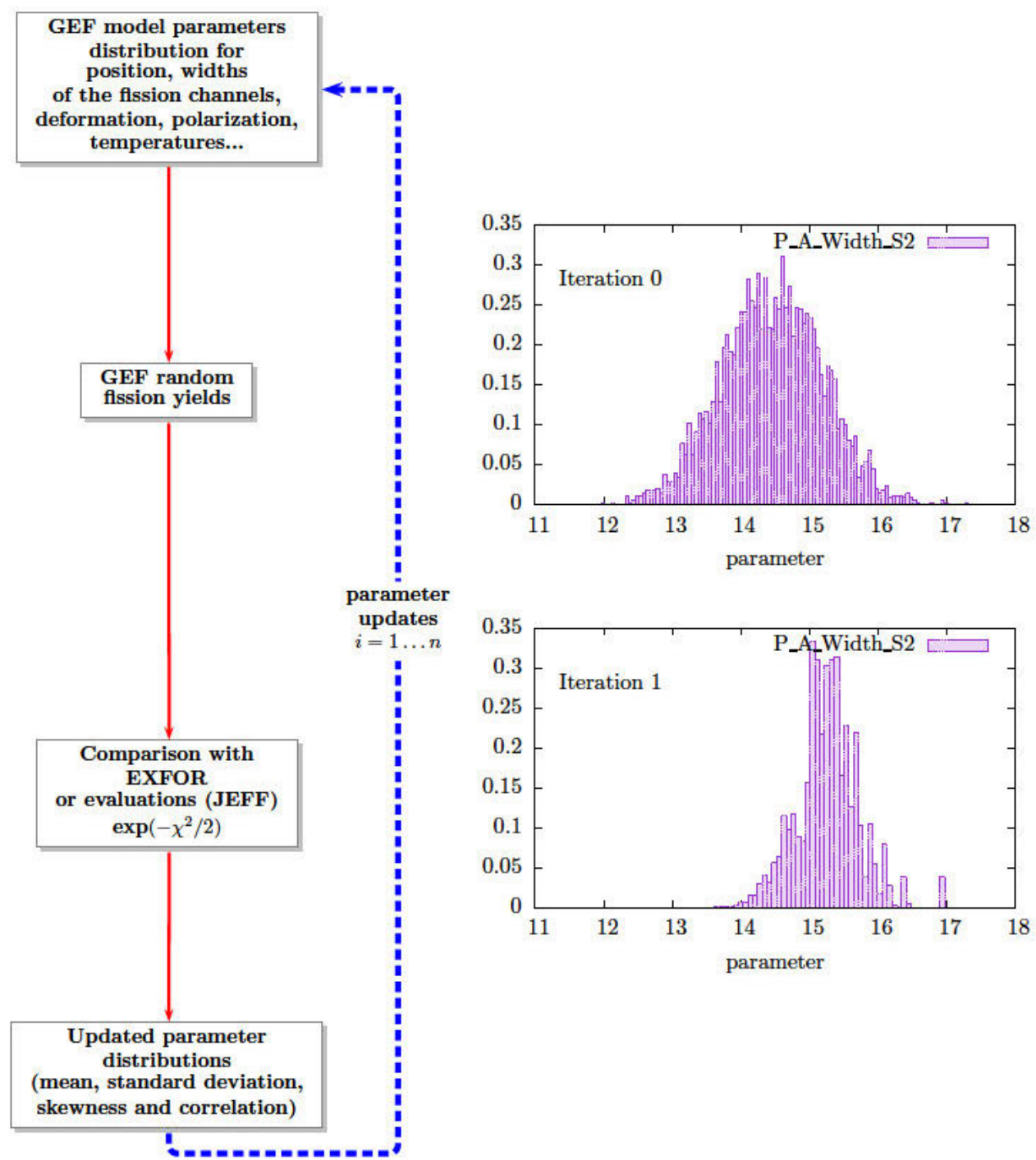

FIG. 2. (Color online) Representation of the Bayesian Monte Carlo in the case of the GEF code for fission yields. On the right are presented the probability distributions for one GEF parameter, along two iterations of the updating process (iteration 0 and 1). In practice, the number of iterations is larger than 1.

In the current XSUSA implementation, the neutron cross sections are varied after the self-shielding calculations, i.e. so-called implicit effects are not taken into account. In other words, it is assumed that cross section perturbations are propagated linearly through the selfshielding calculation. This has the practical advantage that the self-shielding calculations have to be performed only for the calculation case with nominal nuclear data. To verify this simplification, many comparisons have been performed with TSUNAMI [38], which takes into account implicit effects, for multiplication factors, reaction rates, few-group macroscopic cross sections, and reactivity differences in criticality calculations. Good agreement between the uncertainties has been obtained in all cases; examples can be found in Refs. [16, 39]. Nevertheless, the inclusion of implicit effects is under development.
For depletion calculations, another simplification can be applied. For each burn-up step, the self-shielding calculation is performed with nominal isotopic inventories for this specific burn-up step, and not with the actual isotopic inventory originating from the varied nuclear data. This is justified by the fact that the shielded microscopic cross sections resulting from the self-shielding calculations are only little sensitive to the isotopic content of the fuel. When performing the fuel assembly transport calculation, of course macroscopic cross sections are generated with the actual material composition for each individual fuel pin. This proceeding again has the advantage that the self-shielding calculations have to be performed in advance only for one complete depletion calculation with nominal nuclear data, and can be saved for later use in all of the calculations with varied data, leading to a 
TABLE II. List of the 21 most relevant parameters with their uncertainties considered in this work for the ${ }^{235,238} \mathrm{U}$ and ${ }^{239,241} \mathrm{Pu}$ targets. The original table can be found in Ref. [34], Table 7. For more details, see Ref. [34].

\begin{tabular}{|c|c|c|c|}
\hline Parameters & GEF name & $\begin{array}{l}\text { short name in } \\
\text { this paper }\end{array}$ & $\begin{array}{l}\text { Uncertainty } \\
(1 \sigma)\end{array}$ \\
\hline $\begin{array}{l}\text { Position of the shell for the } S_{1} \text { channel } \\
\text { Position of the shell for the } S_{2} \text { channel } \\
\text { Position of the shell for the } S_{3} \text { channel } \\
\text { Position of the shell at } Z \approx 42 \\
\text { Shell effect for the } S_{1} \text { channel } \\
\text { Shell effect for the } S_{2} \text { channel } \\
\text { Shell effect for the } S_{3} \text { channel } \\
\text { Shell at } Z \approx 42 \\
\text { Rectangular contribution to the width of } S_{2} \text { channel } \\
\text { Shell effect at mass symmetry } \\
\text { Curvature of shell for the } S_{1} \text { channel } \\
\text { Curvature of shell for the } S_{2} \text { channel } \\
\text { Curvature of shell for the } S_{3} \text { channel } \\
\text { Curvature of shell at } Z \approx 42 \\
\text { Weakening of the } S_{1} \text { shell with } 82 / 50-N / r m C N / Z_{\mathrm{CN}} \\
(\hbar \omega)_{\text {eff for tunneling of the } S_{1} \text { channel }} \\
(\hbar \omega) \text { eff for tunneling of the } S_{2} \text { channel } \\
(\hbar \omega) \text { eff for tunneling of the } S_{3} \text { channel } \\
(\hbar \omega) \text { eff for tunneling at } Z \approx 42 \\
\text { Width of the fragment distribution in } N / Z \\
\text { Charge polarization }\end{array}$ & $\begin{array}{l}\text { P_DZ_Mean_S1 } \\
\text { P_DZ_Mean_S2 } \\
\text { P_DZ_Mean_S3 } \\
\text { P_DZ_Mean_S4 } \\
\text { P_Shell_S1 } \\
\text { P_Shell_S2 } \\
\text { P_Shell_S3 } \\
\text { P_Shell_S4 } \\
\text { P_A_Width_S2 } \\
\text { Delta_S0 } \\
\text { P_Z_Curv_S1 } \\
\text { P_Z_Curv_S2 } \\
\text { P_Z_Curv_S3 } \\
\text { P_Z_Curv_S4 } \\
\text { T_low_SL } \\
\text { T_low_S1 } \\
\text { T_low_S2 } \\
\text { T_low_S3 } \\
\text { T_low_S4 } \\
\text { HOMPOL } \\
\text { POLARadd }\end{array}$ & $\begin{array}{l}\mathrm{P}_{1} \\
\mathrm{P}_{2} \\
\mathrm{P}_{3} \\
\mathrm{P}_{4} \\
\mathrm{P}_{5} \\
\mathrm{P}_{6} \\
\mathrm{P}_{7} \\
\mathrm{P}_{8} \\
\mathrm{P}_{9} \\
\mathrm{P}_{10} \\
\mathrm{P}_{11} \\
\mathrm{P}_{12} \\
\mathrm{P}_{13} \\
\mathrm{P}_{14} \\
\mathrm{P}_{15} \\
\mathrm{P}_{16} \\
\mathrm{P}_{17} \\
\mathrm{P}_{18} \\
\mathrm{P}_{19} \\
\mathrm{P}_{20} \\
\mathrm{P}_{21}\end{array}$ & $\begin{array}{l}0.1 \mathrm{Z} \text { units } \\
0.13 \mathrm{Z} \text { units } \\
0.1 \mathrm{Z} \text { units } \\
0.12 \mathrm{Z} \text { units } \\
0.2 \mathrm{MeV} \\
0.37 \mathrm{MeV} \\
0.57 \mathrm{MeV} \\
0.12 \mathrm{MeV} \\
0.05 \mathrm{mass} \text { units } \\
0.1 \mathrm{MeV} \\
5 \% \\
5 \% \\
5 \% \\
5 \% \\
10 \% \\
3 \% \\
3 \% \\
3 \% \\
3 \% \\
10 \% \\
0.1 \mathrm{Z} \text { units }\end{array}$ \\
\hline
\end{tabular}

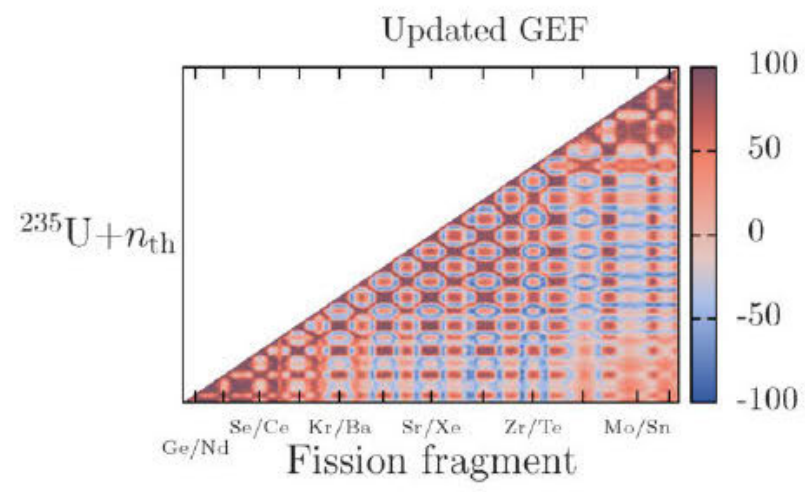

FIG. 3. (Color online) Updated correlation matrices for the fission fragments for ${ }^{235} \mathrm{U}$ with the BMC method. Only half of the symmetric matrices are presented. Correlations are presented in percent (from $-100 \%$ to $+100 \%$ ).

significant performance gain.

XSUSA can also handle fission yield and decay data uncertainties which may be relevant for the uncertainties of nuclide densities in depletion calculations. Such results can be found in Ref. [40]. It was also shown there that fission yield correlations can have a significant influence on the magnitude of the uncertainties of fission product densities, leading to substantial reduction in many cases. However, the methods to generate fission yield correlations are not unique and currently under discussion in the nuclear uncertainty community. Therefore, it was decided to completely omit fission yield uncertainties in XSUSA depletion calculations until generally accepted values for the correlations are available.

Within XSUSA, special emphasis is put on performing sensitivity analyzes for the calculation results; this so far is not yet standard with other sampling based nuclear data uncertainty codes, as described, e.g., in Refs. [21, 41-44]. With the energy-dependent neutron cross sections, for estimating the main contributions to the result uncertainties it is convenient to determine group sensitivities, where typically the nuclear multigroup data for a certain reaction of a certain nuclide are treated as one group. The group sensitivity analysis is performed by determining the squared multiple correlation coefficient $\mathrm{R}^{2}$ as uncertainty importance indicator to quantify uncertainty importance of a group of input variables $\left(\mathrm{X}_{1}, \ldots, \mathrm{X}_{k}\right)$ with respect to an output variable $\mathrm{Y}$. $\mathrm{R}^{2}$ is usually defined as the maximum (squared) simple correlation coefficient between the output variable $Y$ and any linear combination of input variables from the group. It can be computed by the following formula

$$
R^{2}=\left[\rho\left(Y, X_{1}\right), \ldots, \rho\left(Y, X_{k}\right)\right] \times C_{X_{1}}^{-1} \times\left[\begin{array}{c}
\rho\left(Y, X_{1}\right) \\
\vdots \\
\rho\left(Y, X_{k}\right)
\end{array}\right]
$$

Here, $\rho\left(Y, X_{i}\right)$ is the correlation coefficient between the output variable $Y$ and the input variable $X_{i},(\mathrm{i}=1, \ldots, \mathrm{k})$. $C_{X_{1}}^{-1}$ is the inverse of the $(\mathrm{k} \times \mathrm{k})$ - correlation matrix $C_{X_{1}}$ of the group $X_{1}$ of input variables $X_{1}, \ldots, X_{k}$, i.e. the inverse of the matrix of correlation coefficients $\rho_{i, j}=$ $\rho\left(X_{i}, X_{j}\right)$ between all the input variables $X_{i}$ and $X_{j}$, $(i, j=1, \ldots, k)$ from this group. The squared multiple correlation coefficient can be interpreted as the relative amount of output uncertainty coming from the uncertainty of the respective group (i.e. the particular nuclide reaction). 


\section{UPM-SCALE}

This section describes the uncertainty analysis methodology applied at UPM. This analysis is based on Monte Carlo stochastic sampling using the Sampler module along with TRITON depletion sequence from the SCALE6.2 code system [17].

Calculation methodology for uncertainty propagation: SAMPLER sequence

SCALE6.2 includes Sampler super-sequence to assess the uncertainty of a particular response (due to the uncertain knowledge of input data) computed by any SCALE sequence. The workflow can be summarized as follows:

- First, Sampler produces random samples of the input data,

- Then, a large number of calculations of the SCALE sequence of interest are performed, propagating the perturbed data to the specified response,

- Finally, the uncertainty and correlations in the response are obtained by statistical analysis of the output distributions.

Different input data can be perturbed (nuclear data, nuclide concentrations, geometrical dimensions and other model parameters) provided that uncertainties and correlations are known.

Concerning nuclear data, SCALE6.2 includes covariances for multigroup neutron cross-sections, fission product yields and radioactive decay data, so all those parameters can be perturbed, individual or simultaneously. In order to produce the random samples of nuclear data, Sampler uses pre-computed perturbation factors generated by XSUSA code [45] by sampling covariance information. A master file containing perturbation factors for 1000 samples is stored and become available during the Sampler execution. That is, nuclear data parameters are not sampled "on the fly".

In this work, the uncertainty analysis is performed using 200 samples where both multigroup cross-sections and fission yields are perturbed together.

- With respect to cross-sections, perturbation factors for all groups and reactions of all materials were pre-generated by sampling the SCALE6.2 56group covariance library. During Sampler execution, those perturbation factors are read for each sample and a complete set of perturbed problemindependent (infinitely-dilute) multigroup crosssections is computed as well as consistent perturbed parameters required to perform the self-shielding. Those values will be used by TRITON to perform each coupled cross-section processing, transport and depletion calculation.

- With respect to fission product yields, perturbation factors were pre-generated by sampling the independent yields covariance matrix. The yield uncertainties are taken from ENDF/B-VII.1, which are given by fissionable nuclide and for three energies: $0.025 \mathrm{eV}, 0.5 \mathrm{MeV}$ and $14 \mathrm{MeV}$. Only correlations in yields from ${ }^{235} \mathrm{U}$ are included; correlations in yields from other fissionable nuclides are not available. During Sampler execution, the perturbation factors are read for each data sample, and a complete set of perturbed independent yields for all fissionable nuclides and energies is computed. Those values will be read by ORIGEN.

Calculation methodology for depletion: TRITON sequence

TRITON is the SCALE sequence to perform the coupled cross-section processing, transport and depletion calculations. It couples the 2-D discrete-ordinates transport code NEWT and the point depletion and decay code ORIGEN-S using a predictor-corrector approach.

In this approach, cross-section processing and transport calculations are performed at the mid-point of each depletion interval specified by the user, using the isotope concentration computed by predictor depletion steps. Transport results at mid-points (cross sections and flux distribution) are used to perform the ORIGEN-S corrector depletion calculations over the full interval, providing isotopics at the end of the interval. Predictor steps use cross-sections and flux at the mid-point of the previous interval to lead to the transport calculation at the mid-point of the actual depletion interval which will produce updated transport values.

Using TRITON sequence, it is not possible to get both the nuclide composition and $\mathrm{k}_{\infty}$ at the same depletion time, as requested in the benchmark. Consequently, $\mathrm{k}_{\infty}$ at the end of the time interval is obtained by linear interpolation of the computed values at the mid-points. Details of the different calculation steps are given as follows.

Cross-section library

All calculations were performed using the broadgroup cross section library in 56 energy groups from ENDF/B-VII.1 distributed with SCALE6.2 and stored in AMPX master-format. This library, developed for light water reactor physics calculations, was preferred to the fine-group library in 252 energy groups also available in SCALE6.2 based on considerations of computational time and accuracy for current reactors applications.

Cross-section processing

Each perturbed multigroup library containing the problem-independent cross-sections was processed into a problem-dependent library by performing resonance self-shielding. The CENTRM method was used for this task as it is the most rigorous model in both energy and geometry since it performs a continuous energy 1-D transport calculation. The required data for the self-shielding calculations (self-shielding factors and continuous energy cross sections) were also perturbed by using the same perturbation factors computed for 
the infinitely-dilute multigroup cross sections; that way, "implicit effects" are treated in a consistent manner, since all data are based on the same fundamental ENDF/B information.

Different lattice cell calculations were specified for: i) different $\mathrm{U}$ or $\mathrm{Pu}$-enriched rods and ii) rods with the same enrichment but different relative positions in the assembly (e.g. different positions with respect to the water channel and channel box) in order to permit a more accurate prediction of the fuel depletion. Moreover, for the BWR assemblies, accurate Dancoff factors were also calculated using de SCALE/MCDancoff module. The following number of cell calculations was performed for the five considered assemblies: 4 for the PWR $\mathrm{UO}_{2}$ assembly, 5 for the PWR $\mathrm{UO}_{2}+\mathrm{Gd}, 3$ for the PWR MOX, 17 for the BWR $\mathrm{UO}_{2}+\mathrm{Gd}$ and 12 for the BWR $\mathrm{MOX}+\mathrm{Gd}$.

Note the large number of cell calculations required e.g. for the BWR $\mathrm{UO}_{2}+\mathrm{Gd}$ assembly. Only 8 different rod types are found, but since rods with the same composition have different positions with respect to the water channel and the channel box, the corresponding Dancoff factors differ significantly and up to 17 lattice cells were to be specified to accurately catch the effect of the flux distribution on the isotopics and pin-powers along depletion.

In particular, for $\mathrm{UO}_{2}$ and $\mathrm{MOX}$ fuel rods, the LATTICECELL treatment in CENTRM was used. For Gadolinium-bearing fuel rods, a MULTIREGION treatment with five equal-area rings to capture radial depletion of gadolinium was used. In Ref. [46] the impact of the subdivision of $\mathrm{U}+\mathrm{Gd}$ rods was assessed, concluding that while the impact on the isotopic is minimal, on $\mathrm{k}_{\infty}$ is very high in the period when the gadolinium is burning out, being essential the subdivision of the $\mathrm{U}+\mathrm{Gd}$ fuel for criticality calculations.

Transport calculations

In the NEWT transport calculations the order of SN was set to 10; a fine mesh of $4 \times 4$ was used for the square-pitched units and all fuel mixtures and structural materials used P1 scattering whereas all moderator mixtures used P3 scattering. Coarse-mesh finite-difference acceleration option was employed on the global grid to activate a low order solution for homogenized cells in the coarse spatial grid to substantially reduce the computational time to reach convergence.

Depletion and decay calculations

Isotope transmutation and decay calculations were performed with the ORIGEN-S code, which uses a 1-group cross section library. During depletion calculations, only cross sections for isotopes included in the transport calculation are updated on the 1-group library. In order to account for all nuclides having a significant impact on flux, a total of 388 nuclides were added in the transport calculations to update cross sections for ORIGEN-S.

When performing the depletion calculation, materials in the fuel assembly were depleted individually when having different compositions or relative positions in the assembly, to prevent TRITON from averaging the neutron flux in regions with different flux distributions that can impact isotopics. The following number of materials was found adequate for an accurate depletion prediction for the five considered assemblies: 8 for the PWR $\mathrm{UO}_{2}$ assembly, 48 for the PWR $\mathrm{UO}_{2}+\mathrm{Gd}, 3$ for the PWR MOX, 68 for the $\mathrm{BWR} \mathrm{UO}_{2}+\mathrm{Gd}$ and 50 for the BWR MOX $+\mathrm{Gd}$ (those numbers include the 5 different materials considered in the 5 equal-area radial rings per Gd-bearing absorber rod).

A simplification was applied for depletion calculations with a large number of fuel materials to be depleted. In order to decrease calculation run-time in cross-section processing, some materials were grouped together so that a common set of microscopic cross sections were used for all of them, although each material was tracked independently in the depletion calculation.

Normalization was performed using total full assembly power for non-gadolinium rods, and flux for Gd rods. The length of depletion intervals was set to match the burn-up steps requested in the benchmark. Cross-section updates are required more often when properties change rapidly, that is, during poisoning and $\mathrm{Gd}$ depletion. Consequently, finer steps were set at the beginning of the burnup for all assembly types and maintained for larger burnups for assemblies containing Gd rods. Decay calculations were performed by independent ORIGEN-S calculations after the assembly burnup using the requested benchmark steps.

In summary, to propagate uncertainties in nuclear data along depletion, the applied calculation scheme used random pre-computed perturbation factors to generate, for each sample, a perturbed infinitely-dilute multigroup cross-section library, perturbed self-shielding factors and a perturbed yield library for TRITON sequence. Those data were used in an individual coupled cross-section processing, transport and depletion calculation to obtain a sample of the output quantities of interest along burnup. A total of 200 samples were obtained, and it is worth noting that all samples were independent, being the set of perturbed data propagated to downstream codes independently on other runs.

\section{NRG-Fast TMC}

The Fast TMC method can be applied in the case of Monte Carlo transport calculations [19]. It can be explained by a careful examination of how one should combine results of several Monte Carlo runs. This method, although not new, is detailed in the next subsection, to introduce the notation, and to prepare the ground for an explanation of the Fast TMC method. After that, in Subsection II A 5 b, it will be argued that a clever bookkeeping of the individual run results can yield an estimate of the variance due to the differences between these indi- 
vidual runs.

a. Combination of Monte Carlo runs. A Monte Carlo program generates random realizations of a quantity of interest. If the quantity of interest is $x$, the program produces $N$ random realizations $x_{i}$. Using these $x_{i}$, the program does some internal bookkeeping along the way. The program keeps track of the average of the $x_{i}$, and of the average of $x_{i}^{2}$

$$
\begin{aligned}
& S_{1}=\frac{1}{N} \sum_{i=1}^{N} x_{i}, \\
& S_{2}=\frac{1}{N} \sum_{i=1}^{N} x_{i}^{2} .
\end{aligned}
$$

At the end of the run, the program uses the standard Monte Carlo estimators for the average of $x$, which is denoted by $\bar{x}$ here, and for the standard deviation $\sigma$ that goes with it, as defined by

$$
\begin{aligned}
\bar{x} & =S_{1}, \\
\sigma^{2} & =\frac{1}{N-1}\left(S_{2}-S_{1}^{2}\right) .
\end{aligned}
$$

The expectation value for $\sigma^{2}$ is the variance of the variable $x$ divided by $N$

$$
E\left(\sigma^{2}\right)=V\left(\bar{x}^{2}\right)=\frac{V(x)}{N} .
$$

It should be noted that internally the program works with the three variables $N, S_{1}, S_{2}$, but on output it writes the three variables $N, \bar{x}, \sigma$. It is, however, trivial to reconstruct the internal variables, based on the output variables

$$
\begin{aligned}
& S_{1}=\bar{x}, \\
& S_{2}=(N-1) \sigma^{2}+\bar{x}^{2} .
\end{aligned}
$$

Now suppose one has performed $M$ statistically independent runs of the program, each giving output variables $N, \bar{x}_{j}, \sigma_{j}$, with $j=1, \ldots, M$. For the sake of simplicity, it is assumed here that all runs were performed with an equal number $(N)$ of independent realizations of $x$. The generalization of Eq. (9) then reads

$$
\begin{aligned}
S_{1} & =\frac{1}{M} \sum_{j=1}^{M} \bar{x}_{j}, \\
S_{2} & =\frac{N-1}{M} \sum_{j=1}^{M} \sigma_{j}^{2}+\frac{1}{M} \sum_{j=1}^{M} \bar{x}_{j}^{2} .
\end{aligned}
$$

In other words, if one would have performed one large Monte Carlo run with $N \times M$ independent realizations of $x$, the program would have calculated exactly the same $S_{1}$ and $S_{2}$. For this one run, the program would have done it as $S_{1}=(1 / N M) \sum_{i=1}^{N M} x_{i}$, and similarly for $S_{2}$.

Having reconstructed the variables $S_{1}$ and $S_{2}$, one can use Eq. (7) once more. The final Monte Carlo estimator for the combination of $M$ runs of $N$ random realizations is therefore

$$
\begin{aligned}
\bar{x} & =\frac{1}{M} \sum_{j=1}^{M} \bar{x}_{j}, \\
\sigma^{2} & =\frac{1}{M^{2}} \sum_{j=1}^{M} \sigma_{j}^{2}+\frac{1}{N M}\left\{\frac{1}{M} \sum_{j=1}^{M} \bar{x}_{j}^{2}-\left(\frac{1}{M} \sum_{j=1}^{M} \bar{x}_{j}\right)^{2}\right\} .
\end{aligned}
$$

Here we have assumed $N \gg 1$ and $M \gg 1$. The result (11) is a special case (viz. the same $N$ for all runs) of the more general situation described in Ref. [47]. Eq. (11) tells us that it is possible, based on run results $\bar{x}_{j}$ and $\sigma_{j}$, to construct the correct Monte Carlo estimator for the total number $(N \times M)$ of random realizations.

$b$. The variance of the run results. There is, however, more that can be done with the set of $M$ Monte Carlo runs. The expression between curly brackets in the equation for $\sigma^{2}$ in Eq. (11) is in fact the definition of the variance of the $M$ Monte Carlo estimators $\bar{x}_{j}(j=1, \ldots, M)$ denoted as $V(\bar{x})$

$$
E\left(\frac{1}{M} \sum_{j=1}^{M} \bar{x}_{j}^{2}-\left(\frac{1}{M} \sum_{j=1}^{M} \bar{x}_{j}\right)^{2}\right)=V(\bar{x}) .
$$

So far, we have assumed that the $M$ Monte Carlo runs were statistically independent, but otherwise equal. In that case the expectation value for the variance of $\bar{x}_{j}$ is given by Eq. (8), i.e. $V\left(\bar{x}_{j}\right)=V(x) / N$ for all $j$. This is the classical Monte Carlo convergence rate.

The situation changes when the $M$ runs are not identical. When one changes, from one run to the next, not only the random number seed, but also one of the input parameters, the variance of $\bar{x}$ will increase. The variance will reflect the statistical variance as well as the variance due to input parameters variations. A Monte Carlo estimator for this variance, including both statistical and input parameter effects, is

$$
\sigma_{\text {tot }}^{2}=\frac{1}{M} \sum_{j=1}^{M} \bar{x}_{j}^{2}-\left(\frac{1}{M} \sum_{j=1}^{M} \bar{x}_{j}\right)^{2} .
$$

This estimator can be calculated based on the output parameters of the $M$ individual Monte Carlo runs. Moreover, one can also combine the individual Monte Carlo results according to Eq. (11).

The final question is then which part of this total variance is due to statistics, and which part is due to the input parameter variation? This question can be answered by estimating the statistical variance. Eq. (11) is no longer a good estimator for that, because the input parameter variation also influences its results. Instead one can look at the variance of the individual runs, $\sigma_{j}^{2}$. For each individual run, the input parameters are of course fixed, and therefore the variance is due to statistics only. This variance is estimated by the usual Monte Carlo estimator $\sigma_{j}^{2}$. 
A proposal for an estimator of the statistical variance of the combined set of Monte Carlo is then

$$
\sigma_{s t}^{2}=\frac{1}{M} \sum_{j=1}^{M} \sigma_{j}^{2}
$$

The expectation value of this estimator is equal to the expectation value of each of the $\sigma_{j}^{2}$

$$
E\left(\sigma_{s t}^{2}\right)=\frac{V(x)}{N}=V(\bar{x}),
$$

and is therefore suitable as an estimator of the statistical part of the total variance.

Another way to calculate the statistical variance is to repeat the set of $M$ Monte Carlo calculations, this time without input parameter variation. This method doubles the amount of necessary calculation time, though, and one would only like to do this when really necessary. In general, however, it is difficult to know whether this is necessary without performing these calculations explicitly. One could of course use very high numbers of histories (high $N$ ), to get low values for $\sigma_{s t}^{2}$. If these values are much lower than $\sigma_{t o t}^{2}$, than the precise estimate of $\sigma_{s t}^{2}$ is less important for the end result $\sigma_{i p}^{2}$.

On the other hand, there is always the problem with Monte Carlo eigenvalue calculations, that the Monte Carlo estimator for the standard deviation is not entirely correct: the estimator is based on the assumption that there is no correlation between successive neutron batches ('generations'), but in actual fact such correlations do exist. Therefore, the method to repeat the set of Monte Carlo calculations with statistical variation only will be used here as a check on Eq. (15). Further details are given in Subsection IV H 1 b.

Finally, having calculated the total variance using Eq. (13), and the statistical variance using Eq. (15), one can calculate the variance due to the input parameter variation. Here it is necessary to assume that the variance due to statistics and the one due to input parameter variation are independent. This assumption was checked implicitly in the work reported in Ref. [19], through a comparison of several methods, for both high and low values of $M$. The variance due to input parameter variation is then

$$
\sigma_{i p}^{2}=\sigma_{t o t}^{2}-\sigma_{s t}^{2} .
$$

This idea can be applied to any and all input parameters in a Monte Carlo simulation. One can vary one parameter at a time, or many parameters at the same time. The beauty of Monte Carlo is that many parameters can be varied at the same time, while preserving the same convergence rate: in this case, the variance converges as $1 / M$ (the standard deviation as $1 / \sqrt{M}$ ), irrespective of the number of input parameters varied.

Since the amount of nuclear data used in a reactor simulation is very large, the Fast TMC method is well suited for this situation: each nuclear data file contains many parameters that are needed for simulations, such as cross section for various reaction channels, energies and angular distributions of outgoing particles, etc. Therefore this method is applied here to the calculation of local power densities in a full core reactor simulation: with the amount of computer time needed to calculate local power densities in the Martin-Hoogenboom benchmark, it is possible to simultaneously calculate the uncertainties in these local power densities due to the uncertainties in nuclear data.

\section{6. $U U-T M C$}

This section describes the method used at the Uppsala University with DRAGON. The deterministic modeling of the transport of neutrons in a fuel assembly utilizes as material parameters the so-called multi-group microscopic cross-sections as presented earlier. The term multigroup refers to the discretization of the energy variable of such cross-sections and therefore, they are presented as being averaged along different energy bins. One of the most utilized code to compute multi-group microscopic cross-sections is NJOY. It will not only process point-wise information from the different nuclear databases that exist around the world in order to obtain energy averaged parameters, but it also has the capability of arranging the information in different multi-group library formats that can be read by different lattice codes (e.g. WIMS, DRAGON, APOLLO, etc).

The NJOY code, developed at Los Alamos National Laboratory, is modular and sequential. The module that averages the cross-sections in energy and represents them in ENDF format is known as "GROUPR". The ENDF format allows understanding in an easy way the different multi-group information required for an assembly lattice calculation (i.e. microscopic cross-sections, different Legendre orders of the scattering matrices, energy spectrum, released neutrons per fission, etc.). Afterwards, other NJOY modules, such as WIMSR, processes the multigroup ENDF file and arrange it into an ASCII library that some lattice codes understand and may utilize for transport calculations. This final library format is known as WIMS library, and codes such as DRAGON and the ones belonging to the WIMS-family are able to run with the WIMS library format.

As uncertainty analysis has become nowadays part of any physical modeling and code development endeavor, methodologies to propagate (in this particular study) cross-sections uncertainties through lattice codes are needed to be developed in accordance to the library format that a certain code utilizes. Due to the fact that the ENDF multi-group format given by GROUPR is easy to read, understand and manipulate, a new methodology was developed to statistically perturb the GROUPR ASCII file in order to create posterior perturbed WIMS libraries. Thus, as many different WIMS libraries are created, as many times the lattice code will be run in order 
to create a deterministic output into a stochastic one. This is exemplified in Fig. 4.

The module WIMSR reads and manipulates only certain information contained on the ENDF file. Therefore, such information is the one that is required to be statistically perturbed. The information that WIMSR reads from the output of GROUPR is as follows:

1. From the $\mathrm{MF}=3$ data, it reads only the fission $(\mathrm{MT}=18)$ and the capture $(\mathrm{MT}=102)$ crosssections. These will be used to form the total crosssection of the final WIMS library.

2 . From the $\mathrm{MF}=6$ data, it would read only the scattering matrix given by $\mathrm{MT}=2$ correspondent to the fast energy spectrum region. For the resonance and thermal regions, scattering matrices are given by $\mathrm{MT}=221$ (which is computed by the NJOY free-gas model for thermal scattering). It should be noted that the WIMS library format only utilizes $\mathrm{P}_{1}$ and $\mathrm{P}_{0}$ terms for moderator nuclides. For other nuclides such as actinides, etc., only the $\mathrm{P}_{0}$ matrix is considered. The energy-integration of both $\mathrm{MT}=2$ and $\mathrm{MT}=221$ will be used to compute the elastic scattering part of the total cross-section.

3. From the $\mathrm{MF}=6$ and $\mathrm{MT}=50-91$, WIMSR will add them per energy group in order to form inelastic scattering and thus, after energy integration, update the total cross-section.

4. From $\mathrm{MF}=6$ and $\mathrm{MT}=18$, the Nu-Fission crosssection is taken.

5. Even though the $\mathrm{MF}=5$ data contained in the ENDF file gives the fission spectrum, the WIMS library format fixes the fission spectrum and thus, does not take into account these info from the ENDF file.

Now, the uncertainty analysis would perturb ALL terms of the parameters that are required for the transport calculation. This is how the perturbation methodology works in order to conserve any further transport calculation:

1. Covariance data is given to the total energy integrated terms of the multi-group cross-sections. From this information (which can be obtained from the ERRORJ module of NJOY) the fission and capture cross-sections can be perturbed. It was realized that if the fission cross-section $(\mathrm{MF}=3, \mathrm{MT}=18)$ is perturbed in the ENDF ASCII file, the module WIMSR will change not only the total cross-section but also Nu-fission. This is a good thing since perturbations to the fission cross-section are being conserved by WIMSR. Also, any perturbation to the capture cross-section will immediately update the total cross-section as well.
2. $\mathrm{MF}=6, \mathrm{MT}=2$ and 221 should be statistically perturbed in order to change the elastic scattering matrices and also the total cross-section. Since only the $\mathrm{P}_{0}$ covariances are given, then only the $\mathrm{P}_{0}$ matrix can be perturbed.

3. For the inelastic scattering term, the perturbation was done only at the MT=91 data. This is good enough since WIMSR adds up all the MT=51-91 terms and thus, the propagation of the perturbation is carried out correctly.

4. The Nu-fission term $(\mathrm{MF}=6, \mathrm{MT}=18)$ was modified in a way that only the $\nu$ uncertainty was propagated, since the fission perturbation is carried out at the $\mathrm{MF}=3, \mathrm{MT}=18$ data.

5. For the WIMS library, even if $\mathrm{MF}=5$ data could be perturbed in order to change the fission spectrum, no effect whatsoever would have on the spectrum of the final WIMS library. This is the only term that cannot be perturbed in the ENDF file and thus, such perturbation should be carried out at the final WIMS library.

\section{UKAEA-FISPACT-II}

Provided with the reactor burn-up simulation data from the lattice code CASMO-5, FISPACT-II [18] was modified to also accommodate the fine 586-group neutron spectrum data with newly ENDF/B-VII.1 processed data and CALENDF-generated [48] probability tables for self-shielding corrections. FISPACT-II possesses a new POWER keyword which causes re-normalisation of the total flux to match a specified $\mathrm{W} / \mathrm{cc}$ volumetric power to a defined set of fissile isotopes. The normalisation uses isotopic KERMA values provided by collapsing of the neutron spectrum with KERMA data from the nuclear data files, which can be specified using any appropriate $\mathrm{mt}$ values (for example the total $\mathrm{mt}=301$, non-elastic $m t=303$, inelastic $m t=304$, fission $m t=318$, disappearance $m t=401$, photons $m t=442$, total kinematic $\mathrm{mt}=443$ or any combination thereof). To best match the self-shielding factors (SSF) for fission and ${ }^{238} \mathrm{U}$ capture from CASMO-5, the probability tables were applied as energy-dependent SSF for each group based on total cross-section dilution self-shielding (rather than using macro-partial SSF, a more sophisticated approach available to FISPACT-II).

As shown in Fig. 5, the energy-dependent reaction rates for BOL SF97 require virtually no self-shielding factors for energies below $10 \mathrm{eV}$ (due to the fine group resolution). Above $10 \mathrm{eV}$, the ${ }^{235} \mathrm{U}(\mathrm{n}, \mathrm{f})$ has some $\mathrm{SSF}$ correction which is not significant for the overall reaction rate. The ${ }^{238} \mathrm{U}$ capture is quite different, with significant resonances contributing to the reaction rate up to the keV energy region. Group SSF range down to 0.02, and average to an effective SSF around 0.1-0.2, which evolves 

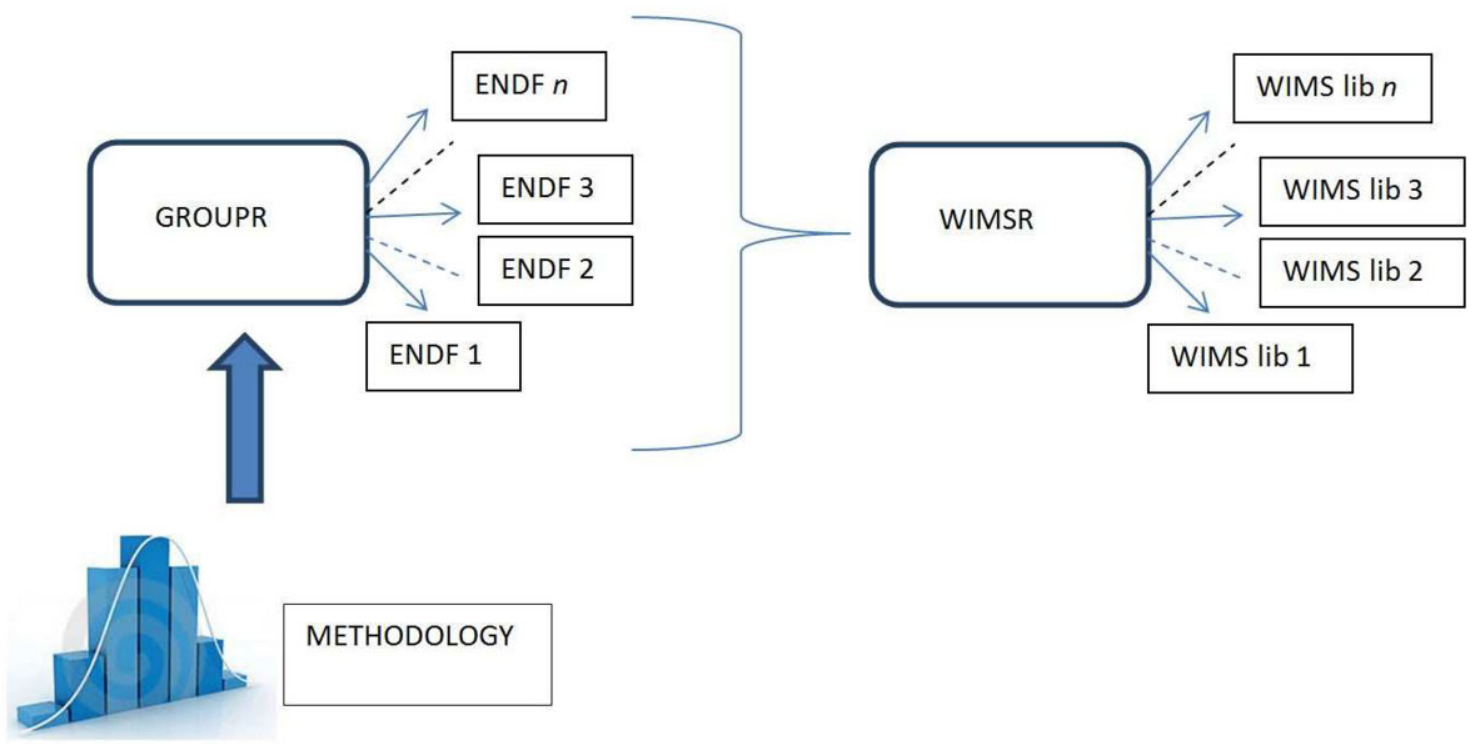

FIG. 4. (Color online) Processing steps for the Uppsala methodology to propagate DRAGON libraries.

through burn-up. The plutonium production rate is extremely sensitive to these SSFs, which dictate the fission ${ }^{235} \mathrm{U} /{ }^{239} \mathrm{Pu}$ ratio and composition throughout the fuel lifetime. For this analysis, SSFs are updated for each change in spectrum, as reaction rates are re-calculated for each of these steps.

For this uncertainty quantification exercise, the TotalMonte Carlo fission yield uncertainties on the thermal ${ }^{235} \mathrm{U},{ }^{239} \mathrm{Pu}$ and ${ }^{241} \mathrm{Pu}$, as well as $400 \mathrm{keV}{ }^{238} \mathrm{U}$ are quantified through use of random fission yield files. 500 neutron-induced fission yield files, which are generated by input parameter variation using the GEF-4.2 fission simulation code (as presented in section II A 2), were used for multiple simulations. Simulations for each case are performed in parallel and code outputs are statistically collapsed to determine moments for nuclide inventories, heat outputs, activity, etc. The tremendous advantage of this approach is that all covariances are effectively treated through sampling of physically-consistent input physics, without the need for complex covariance methods or pathway analyzes. An example of these simulations is shown in Fig. 6. A correlated uncertainty has also been calculated, using the variation in collapsed covariance uncertainty with variation in nFY files. This coupled contribution is typically less than $10 \%$ of the reaction rate collapsed-covariance uncertainties.

Uncertainty in an integral quantity such as decay heat hides a tremendous amount of information, since dozens or hundreds of nuclides each contribute similar fractions of the total quantity for most cooling times - particularly for times not long after irradiation. Since FISPACTII also handles all independent fission yields, decays and reaction channels [49-51], the inventory is handled in full. The use of cross section uncertainties, as provided by covariance files, requires identification of each combination of parents, reactions, decays and loops which result in the production of every nuclide of interest. TMC analyses can be performed without these combinatorially complex tasks, providing uncertainties on all nuclides simultaneously as shown in Fig. 7.

Note the exceptionally low uncertainties for nuclides within the fission yield peaks, which are not realistic for many. This feature is a direct consequence of the effect of limited range of values generated by default parameter variation within $\mathrm{GEF}$, which must be modified to generate experimentally-faithful yield variation. Nuclides with relatively small yields, approximately those below 1 per $10^{5}$ fissions, have an increased uncertainty which is an artifact due to statistical variation inherent to the MonteCarlo GEF method.

FISPACT-II employs a sophisticated reaction rate uncertainty quantification method using available cross section covariances and deep reaction/decay pathway analysis [52]. All independent fission yields, open reaction channels and decays are considered, following from hundreds to a few thousand nuclides. FISPACT-II can process ENDF-6 NI-type covariance data with $\mathrm{LB}=1,5$ or 6 , using a projection operation to match covariance and cross-section energy grids, which are stored in multigroup format (586-group for CASMO-5 based simulation). Once individual reaction rate uncertainties are calculated for a given incident particle spectrum, the reaction/decay production pathway for each nuclide must be determined. In the Takahama case a typical production route for ${ }^{137} \mathrm{Xe}$, a precursor to ${ }^{137} \mathrm{Cs}$, the path is broken 

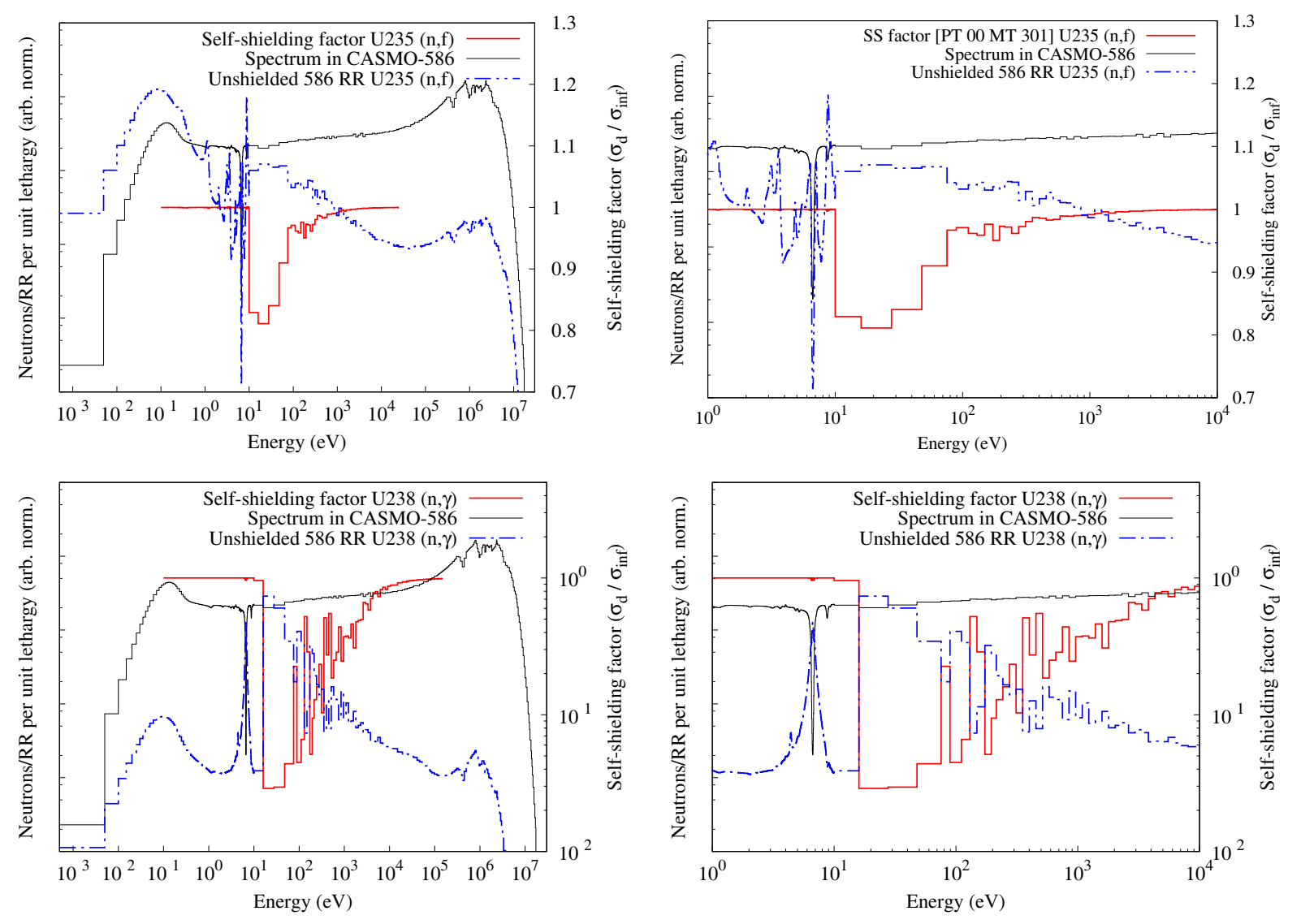

FIG. 5. (Color online) FISPACT-II collapsed energy-dependent unshielded reaction rates for ${ }^{235} \mathrm{U}(\mathrm{n}, \mathrm{f})[\mathrm{top}]$ and ${ }^{238} \mathrm{U}(\mathrm{n}, \gamma)$ [bottom], with probability table self-shielding factors superimposed. The ultra-fine energy resolution with the CASMO-5 586 group eliminates the need for self-shielding below $10 \mathrm{eV}$, but for ${ }^{238} \mathrm{U}$ capture the factors above $10 \mathrm{eV}$ are significant, resulting in an effective SSF around 0.1-0.2.

down through fission of $29 \%{ }^{235} \mathrm{U}, 46 \%{ }^{239} \mathrm{Pu}, 14 \%$ ${ }^{241} \mathrm{Pu}$ and $8 \%{ }^{238} \mathrm{U}$, with varying contributions through ${ }^{137} \mathrm{I},{ }^{137} \mathrm{Te}$, isomeric and direct production. Some minor contributions from other fission and paths including reactions are also analyzed in FISPACT-II. Uncertainties for each reaction are then calculated and weighted to determine a final nuclide inventory uncertainty.

For fission scenarios, this can be extremely complex due to combinatorial growth, requiring intelligent path 'pruning'. This employs minimum path/loop values for inclusion and maximum path depth values, which have been set to defaults except where proven insufficient. Both the fission yield and reaction rate uncertainties are calculated together, so that the uncertainties are propagated together. For each simulation with varied fission yield files, the reaction rate uncertainty is also calculated. While the uncertainty in the effective cross section for each reaction channel in each time step does not vary in each of these simulations, the fraction of each pathway does. Difference uncertainties between, say, the ${ }^{235} \mathrm{U}$ or ${ }^{241} \mathrm{Pu}$ fission rates and yields are coupled, so that the reaction rate uncertainty will vary as the ratio is changed. Exam- ples are shown in Fig. 8, with varied dominant nuclide sets, TMC-based nFY uncertainty, covariance reaction rate uncertainty and their correlated contributions.

Note that the uncertainty files $\mathrm{mf}=33$ for the major nuclear data libraries do not contain uncertainties within the resolved resonances or below, which completely discards any uncertainty quantification for reactions where those energies are significant. This is precisely the case for neutron capture and non-threshold fission, which plays a central role in thermal reactor physics. Without these data all uncertainties are guaranteed to be underestimates.

\section{SCK Fission Yields}

SANDY $[53,54]$ is a new NJOY-wrapping numerical code developed at SCK-CEN to perform the sensitivity analysis (SA) and uncertainty propagation (UQ) of nuclear data. Amongst its capabilities, such code generates covariance matrices for independent fission yields using a generalized least-square (GLS) approach. After collecting 


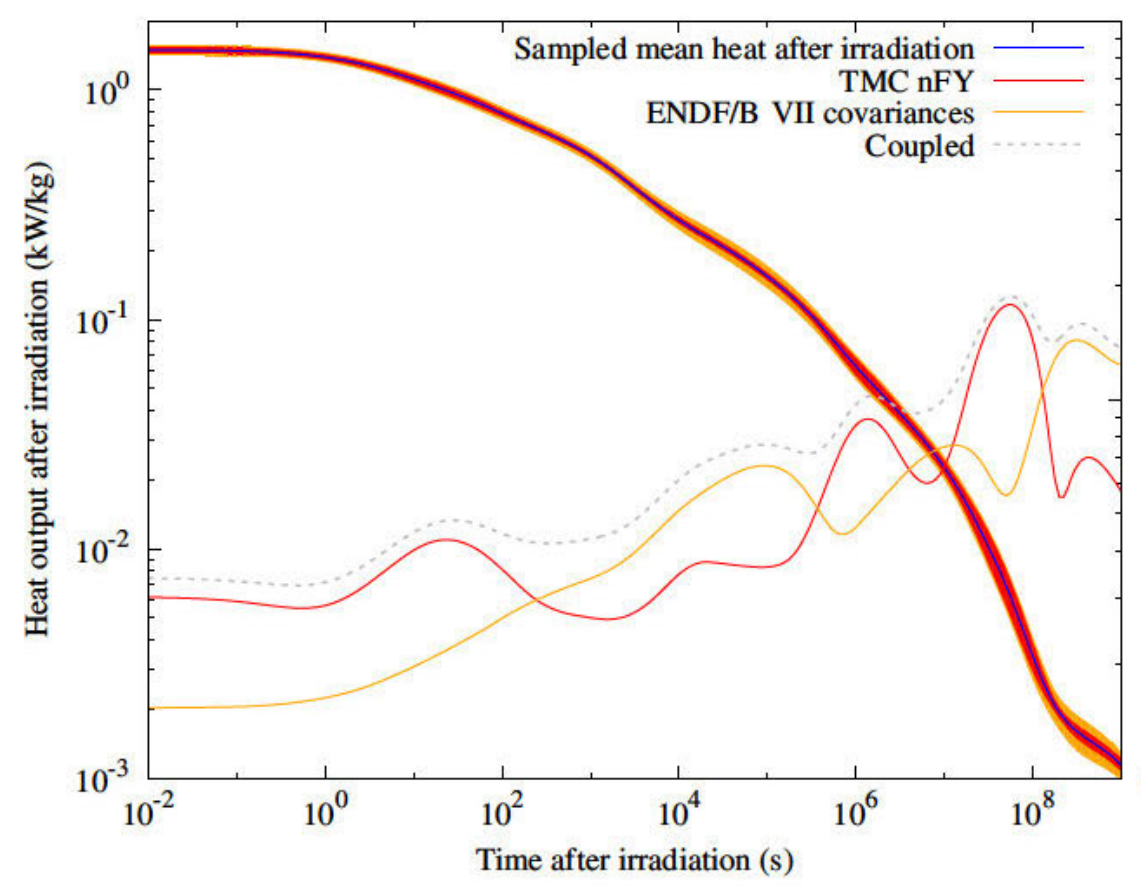

FIG. 6. (Color online) Example decay heat from Takahama SF97-1 after 45 MWd/TU, using ENDF/B-VII.1 nuclear data file covariances for reaction rate uncertainty and TMC thermal neutron sampling for yield uncertainty. The coupled combination includes both, with an additional correlation due to variation in the collapsed covariance uncertainty with $\mathrm{nFY}$ variation. The uncertainty bands have been multiplied by 10 to aid visualization on log-log axes.

best-estimate and uncertainty values from the ENDF-6 files, such data are constrained to comply with the conservation equations of a fission event e.g. conservation of mass, charge and number of fission fragments, symmetry, correlation between independent and chain fission yields.

Hence, covariance matrices are produced and independent fission yields are upgraded. Such an update tackles the need for more consistency of fission yield uncertainties in the major general purpose libraries (JEFF, ENDF/B) $[12,13,26]$, which only loosely abide by the conservation laws at the current stage. Then, given an upgraded array of best-estimate independent fission yields $Y$ and a covariance matrix $C$, random samples are drawn from a multivariate normal probability density function (pdf) $N(Y, C)$ using a standard Monte Carlo sampling procedure. The new random sets of independent fission yields replace the original values in "perturbed" ENDF-6 files. In each "perturbed" file, cumulative fission yields are also recalculated using the random independent yields and the Q-matrix equation which involves the use of radioactive branching ratios [55].

In this work, we created "perturbed" files for the U and $\mathrm{Pu}$ fissioning systems of ENDF/B-VII.1. Additionally, the perturbation of the Q-matrix is performed and an extra set of files with perturbed "fission yields and branching ratios" is made. The fission yields from SANDY are used in this work with CASMO-5 as an option in the SHARK-X approach.

\section{B. Considered Systems}

In the following, details for the different assembly systems are given and are summarized in Table III. Then, the Martin-Hoogenboom simplified full core is presented.

\section{1. $\quad \mathrm{PWR} \mathrm{UO}_{2}$ Assembly}

The 2-dimensional assembly model for the PWR with $\mathrm{UO}_{2}$ fuel is the Three Mile Island Unit 1 (TMI-1) type. The specifications for such assembly model can be found in Refs. $[56,60]$. The general characteristics are reproduced here and in Table III.

The TMI- 1 assembly is a $\mathrm{UO}_{2}$ fuel assembly with a $15 \times 15$ lattice of 212 fuel rods and is represented in Fig. 9 .

The number of guide tubes is 16 (in light blue in Fig. 9 left), with one instrumental tube at the center of the assembly. Four fuel rods contain a small amount of gadolinium (2 w\%), which is believed to have a small impact on the assembly characteristics. The number of $\mathrm{UO}_{2}$ fuel rod (without $\mathrm{Gd}$ ) is 204, with an enrichment in ${ }^{235} \mathrm{U}$ of $4.12 \mathrm{wt} \%$. Some specific geometrical data are given in Table IV. For the presented burn-up calculations, the limit in the burn-up value is $40 \mathrm{GWd} / \mathrm{tHM}$. 